\title{
Cierre de leak periprotésico mitral con Amplatzer Vascular Plug III, vía transapical y toracotomía mínima
}

Dante Lindefjeld, Alejandro Martínez, Felipe Heusser, Ricardo Zalaquett, Samuel Córdova, Manuel Méndez, Pedro Becker.

Departamento de Enfermedades Cardiovasculares. Pontificia Universidad Católica de Chile.

\section{Introducción}

La dehiscencia parcial de una válvula cardíaca protésica, produciendo una fuga perivalvular (FPV), es una complicación que puede ocurrir en el seguimiento alejado de los pacientes sometidos a reemplazo valvular. Es clínicamente significativa en 2 a $5 \%$ de los casos, en los que produce insuficiencia importante o hemólisis. La etiología corresponde a una pérdida de continuidad de las suturas, ya sea por la técnica empleada, por endocarditis o por calcificación anular. ${ }^{1,2}$

La reoperación es el gold estándar en el manejo de la FVP, pero está asociada a una alta morbi-mortalidad, especialmente cuando el paciente es añoso, tiene múltiples comorbilidades o fue operado más de una vez. ${ }^{3}$ En los casos donde el riesgo quirúrgico es demasiado alto, se ha planteado el cierre endovascular percutáneo, para lo que se han reportado con éxito pequeñas experiencias que utilizan dispositivos oclusores Amplatzer. Estos procedimientos percutáneos, sin embargo, son especialmente complejos en el tratamiento de los defectos en prótesis mitrales, donde existe una alta tasa de fracaso. La dificultad radica en que para acceder al sitio de la FPV de una prótesis mitral, se requiere un largo y sinuoso recorrido por vía anterógrada transeptal o retrógrada transaórtica, con la resultante dificultad para traspasar el defecto y para obtener el apoyo necesario, cuando se ha traspasado. En esta publicación reportamos el resultado exitoso de tres pacientes con FPV mitral tratados con el implante de un dispositivo Amplatzer, a través de punción directa del ápex cardíaco.

\section{Métodos:}

\section{Pacientes:}

Se trataron tres pacientes de sexo femenino, con FVP de prótesis mitral, que en todas era Saint Jude. Se presentaron con anemia hemolítica e insuficiencia valvular, y con insuficiencia cardíaca capacidad funcional III - IV (NYHA). Sus edades eran 63, 61 y 72 años. Todas tenían 3 cirugías cardíacas previas por reemplazo valvular, y se consideraron de alto riesgo quirúrgico actual. Dos de ellas tenían además prótesis valvular aórtica. La ubicación de la FVP era posterior o medial (P2, A2-A3 y A3; Fig 1 y 2). En 2 casos se intentó previamente el cierre percutáneo utilizando la vía anterógrada transeptal o transaórtica, sin lograr retrogradar el defecto. En la otra paciente se indicó directamente el acceso transapical, debido a la localización medial de la FVP y la presencia de una válvula protésica aórtica.

\section{Técnica empleada:}

En los tres casos la FVP mitral fue cerrada utilizando un dispositivo Amplatzer Vascular Plug III (Fig 3), vía transapical, en un pabellón quirúrgico, y guiado mediante ecografía transesofágica (ETE) y radioscopía. Las pacientes fueron premedicadas con cefazolina endovenosa. Bajo anestesia general, con intubación bronquial selectiva (doble lumen), se practicó una tora-

Correspondencia:

Dr. Dante S. Lindefjeld Calabi.

División de Enfermedades Cardiovasculares,

Escuela de Medicina, Pontificia Universidad Católica de Chile,

Marcoleta 367, 2do piso, Santiago - Chile.

E - mail: dslindef@puc.cl 


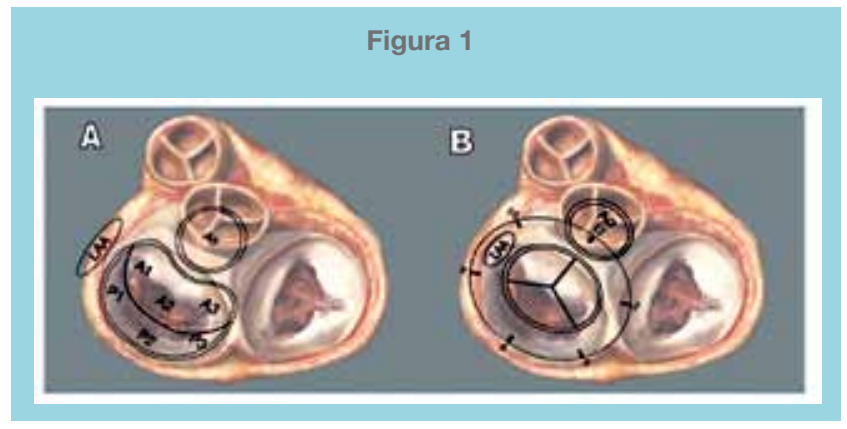

Figura 1. Se muestra la segmentación y nomenclatura utilizada para la localización de los defectos para-protésicos. En "A” observamos la clasificación de Carpentier, inicialmente aplicada para velo y borde libre de los mismos, en este caso segmentado el perímetro protésico. En “B” se detalla la segmentación del anillo protésico o del anillo valvular guiados por el huso horario, adoptando como 12 la proyección de la válvula aorta (Ao) y las 9 la proyección de la orejuela izquierda (LAA).

Fig 3: Amplatzer Vascular Plug III.

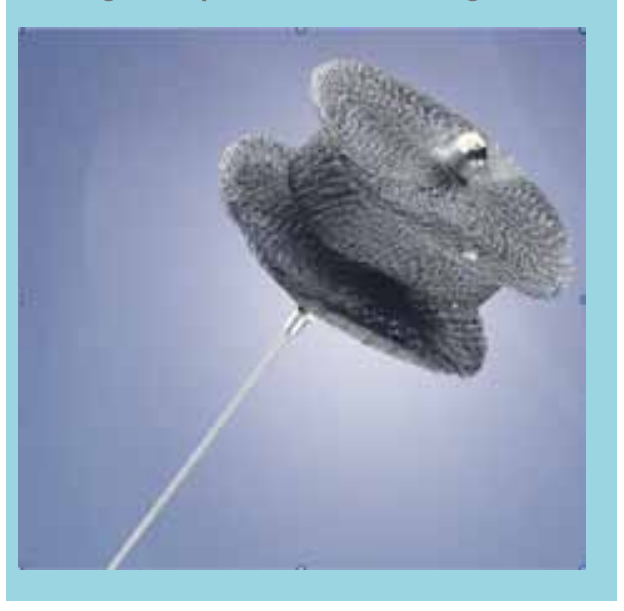

cotomía izquierda mínima, eligiendo el espacio intercostal adecuado con el apoyo de ETE y radioscópico. Posteriormente se expuso el ápex cardíaco (ventilando sólo el pulmón derecho, para evitar interferencia de la língula pulmonar izquierda durante la punción) y con visión ecocardiográfica transesofágica, se efectuó la punción y colocación de una vaina de 6 French (técnica de Seldinger) en el ápex del corazón, previa colocación de jareta de puntos corridos alrededor del sitio de punción (Fig 4 y 5). Enseguida, se orientó la vaina hacia el sitio de la FPV, que se atravesó con relativa facilidad usando una guía glide 0.035 pulgadas. A través de esta guía, habiendo retirado la vaina, se avanzó el catéter de entrega del dispositivo Amplatzer para la liberación del vascular plug III, exactamente en el sitio de la FPV (Fig 6 y 7). El tamaño del dispositivo se

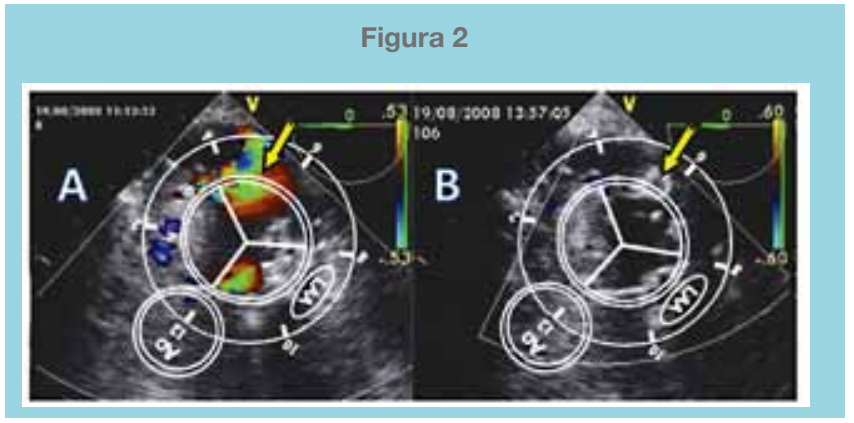

Figura 2. Se muestran dos momentos del procedimiento del cierre de un defecto o fuga para-protésica mitral. La proyección ecocardiográfica corresponde a un eje corto de la base del corazón obtenida en posición transgástrica. En ambas destaca la utilización de la segmentación del perímetro anular o protésico con la aplicación del "huso horario"; las 12 corresponde a la proyección de la válvula aórtica (Ao). En " $A$ ” la flecha amarilla muestra la posición del defecto localizado con ayuda del Doppler color. Defecto de situación POSTERIOR. En “B”, siempre con ayuda del Doppler color, se observa la ausencia de flujo a nivel del defecto; este se encuentra ocluido por un Amplatzer señalado por la flecha amarilla.

\begin{tabular}{|c|c|c|c|c|c|c|c|}
\hline \# & $\begin{array}{l}\text { Edad } \\
\text { años }\end{array}$ & $\begin{array}{l}\text { Cirugías } \\
\text { previas }\end{array}$ & $\begin{array}{l}\text { Sitio Leak } \\
\text { mitral }\end{array}$ & $\begin{array}{l}\text { Intento } \\
\text { percutáneo } \\
\text { previo }\end{array}$ & $\begin{array}{l}\text { Amplatzer } \\
\text { Vascular } \\
\text { Plug III }\end{array}$ & $\begin{array}{c}\text { Tiempo } \\
\text { Radioscopía } \\
\text { (PATM) }\end{array}$ & Complicaciones \\
\hline 1 & 63 & 3 & P2 & Transeptal & $6 / 3 \mathrm{~mm}$ & 01:03:31 & no \\
\hline 2 & 61 & 3 & A2-A3 & $\begin{array}{l}\text { Transeptal y } \\
\text { Retrogrado }\end{array}$ & $8 / 4 \mathrm{~mm}$ & $00: 32: 58$ & no \\
\hline 3 & 72 & 3 & A3 & no & $10 / 5 \mathrm{~mm}$ & $00: 31: 15$ & $\mathrm{Si}^{*}$ \\
\hline
\end{tabular}

eligió de acuerdo al tamaño del defecto. Comprobada la buena posición del dispositivo, se retiró el catéter de entrega y se procedió a cerrar la jareta y la toracotomía por planos. Los detalles técnicos se describen en la tabla 1 .

En las tres pacientes fue factible el cierre de las FVP mitrales. Mientras que en dos casos no hubo interferencia con la movilidad de los discos de la válvula, en una de ellas se observó disminución de la movilidad de un hemidisco posterior al implante del dispositivo, pero sin causar impacto funcional significativo sobre la válvula (mínima gradiente transvalvular).

No hubo complicaciones durante el seguimiento intrahospitalario. Al mes del procedimiento no se registraron nuevas caídas del hematocrito, y la capacidad funcional mejoró sustancialmente en las tres pacientes. 


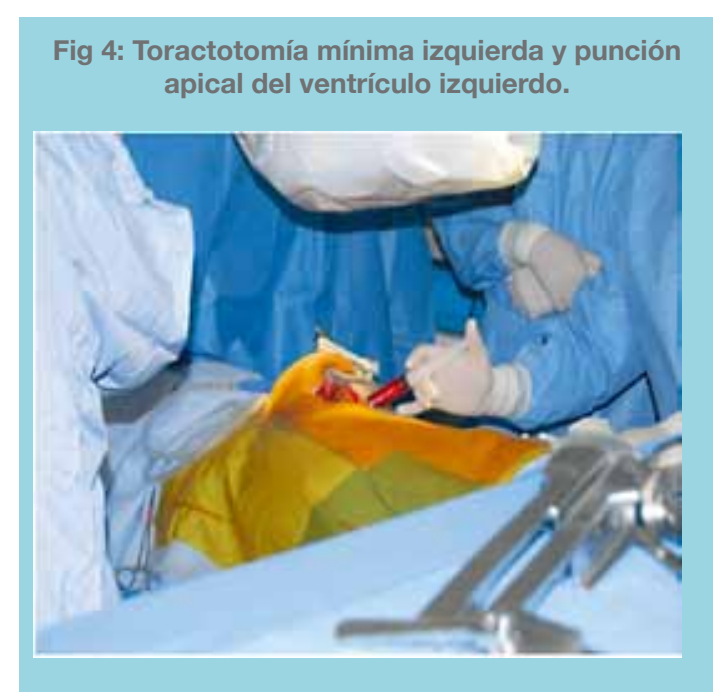

Figura 6: visión de ETE del implante del dispositivo.

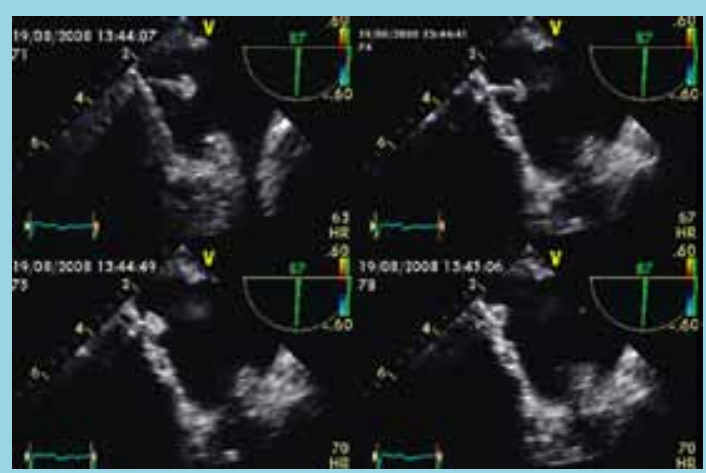

Figura 7: Observación con Doppler color a 0 y $90^{\circ}$ pre y post implante.

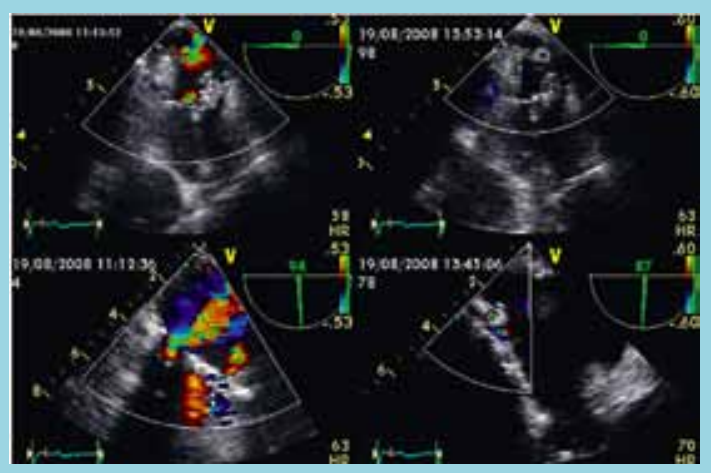

\section{Discusión:}

La decisión de reparación quirúrgica de fugas (leaks) valvulares periprotésicas (FVP), con repercusión hemodinámica o hematológica, suele ser compleja, especialmente cuando los pacientes son de alto riesgo, ya sea por la presencia de co-morbilidades asociadas o cirugías cardíacas previas.

En estos pacientes, el cierre percutáneo con distin-
Fig 5: Catéter introductor de 6 Fr en el ápex cardiaco.
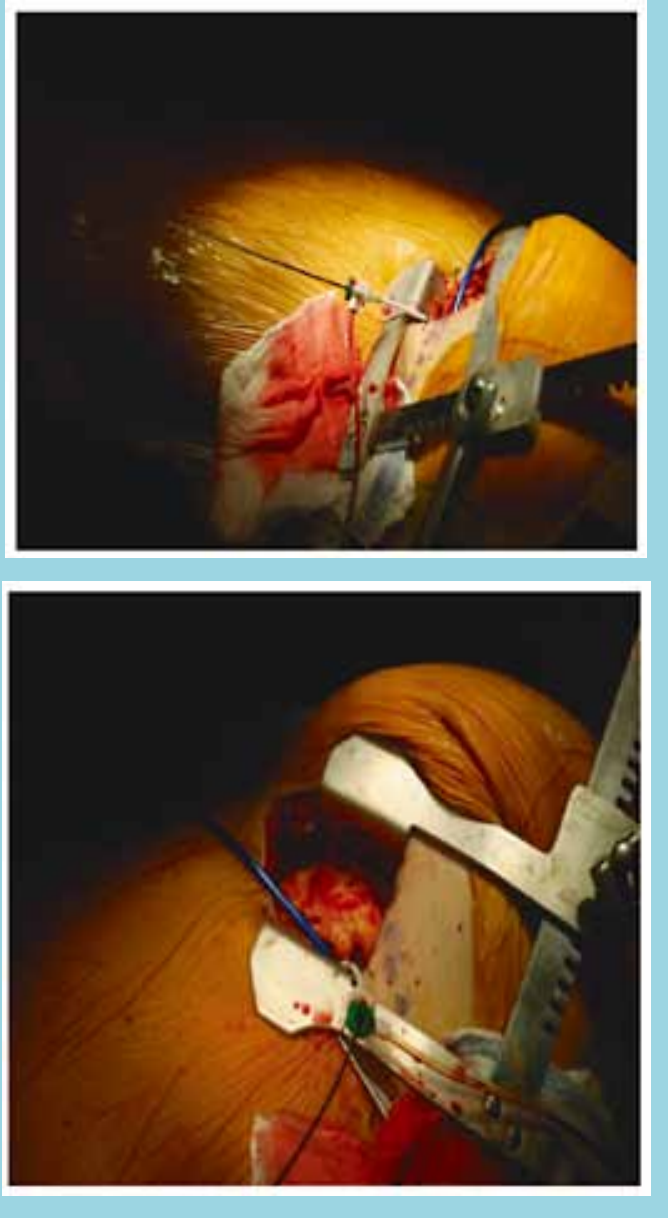

tos dispositivos ha generado creciente interés. Es así que en el año en 1992 se reportó el cierre exitoso de dehiscencias periprotésicos aórticos con el sistema de doble paraguas Rashkind. ${ }^{4}$ Posteriormente surgieron reportes de cierre tanto para defectos aórticos como mitrales con coils, oclusores vasculares de GianturcoGrifka, y en la actualidad dispositivos Amplatzer, en sus diferentes diseños ${ }^{5-9}$, incluidos aquellos inicialmente diseñados para el cierre de defectos del tabique interauricular ${ }^{10}$, con los cuales se ha podido resolver exitosamente casos de fugas valvulares periprotésicas aórticas y mitrales.

La elección del tipo y tamaño de dispositivo a utilizar es de suma importancia, con la finalidad de poder cerrar íntegramente el defecto, evitar su migración o interferir en el funcionamiento normal de la prótesis valvular. ${ }^{11}$ No existe un dispositivo específico fabricado para esta finalidad, pero dentro de la gama de diseños, los Vascular Plug van ganando mayor aceptación. ${ }^{12,13}$ En nuestro reporte, describimos el uso del Vascular Plug III, ya que la forma ovalada de sus discos y de su rodete, permiten un mejor posicionamiento y adecua- 
ción morfológica del dispositivo sobre el defecto, que habitualmente tiene esta forma.

La vías de acceso descritas para el cierre percutáneo son la retrograda (transaórtica) o anterógrada (por punción transeptal auricular). Existe predilección por la vía retrograda para el cierre de dehiscencia mitral, ya que permite el pasaje del alambre guía en la misma dirección del flujo del jet regurgitante y permite apoyo suficiente para posicionar el sistema de entrega Amplatzer, a la inversa de lo que ocurre con la vía anterógrada o transeptal, que a su vez, tiene como inconvenientes la posición del defecto, en especial si es en el borde septal, lo que condiciona apoyo insuficiente de las guías y sistema de entrega Amplatzer. También entorpece o imposibilita el procedimiento la existencia de válvulas protésicas en posición aórtica, situación presente en dos de nuestras tres pacientes tratadas.

Estos procedimientos demandan paciencia, habilidad técnica de los operadores, y generalmente duran varias horas tanto de procedimiento como de radioscopía. En ocasiones, pese a múltiples esfuerzos por acceder al defecto ya sea por vía retrógrada o anterógrada, el procedimiento resulta infructuoso. ${ }^{14,15,16,17}$

Existen reportes exitosos, resueltos por punción ventricular apical, vía torácica percutánea y anestesia general. Este acceso facilitó un mejor direccionamiento del catéter y pasaje del alambre guía a través del defecto, menor tiempo de procedimiento y radioscopia. Aunque es una alternativa novedosa, no está exenta de complicaciones graves, tales como hemo o neumotórax, tamponamiento, etc. ${ }^{18,19,20}$
Debido a esto nos pareció apropiado emplear la técnica de mini toracotomía izquierda (sin circulación extracorpórea) y punción apical directa, descrita para el implante de válvulas aórticas de Cribier - Edwards (diseñadas para implante percutáneo). De esta manera es posible controlar el sitio de punción apical y evitar complicaciones. ${ }^{21,22}$

Creemos que es indispensable contar con visión ecocardiográfica transesofágica, ya que aporta información valiosa, tanto al elegir el sitio de punción apical, definir la localización del defecto y guiar el pasaje del alambre guía como del sistema de entrega del Amplatzer. Finalmente, este método asegura el posicionamiento del dispositivo, detecta posibles interferencias con la válvula protésica, y permite evaluar el resultado final de procedimiento. ${ }^{23}$

\section{Conclusión:}

En nuestro reporte de 3 casos, el cierre de FVP mitral con un dispositivo específico fue exitoso, demostrando que cuando la vía percutánea no es posible, el acceso por vía transapical y toracotomía mínima es una alternativa atractiva.

Por lo tanto, cuando los intentos de cierre de FVP por vía percutánea sean infructuosos o imposibles, alentamos al equipo de intervencionistas, cirujanos cardíacos y ecocardiografistas, a emplear la vía transapical con toracotomía mínima, guiados bajo visión ecocardiográfica transesofágica, empleando el dispositivo que mejor se ajuste al defecto a tratar.

\section{Referencias:}

1. REMADI JP, BIZOUARN P, BARON O, AL HABASH O, DESPINS P, MICHAUD JL, et al. Mitral valve replacement with the St. Jude medical prosthesis: A 15 year follow up. Ann Thorac Surg 1998; 66: $762-7$.

2. JINDANI A, NEVILLE EM, VENN G, WILLIAMS BT. Paraprosthetic leak: A complication of cardiac valve replacement. J Cardiovasc Surg (Torino) 1991; 32: 503 -8.

3. POTTER DD, SUNDT TM. Risk of repeat mitral valve replacement for failed mitral valve prostheses. Ann Thorac Surg 2004; 78: $67-72$.

4. HOURIHAN M, PERRY SB, MANDELL VS, ET AL. Transcatheter umbrella closure of valvular and paravalvular leaks. J Am Coll Cardiol 1992;20:1371-1377.
5. EISENHAUER AC, PIEMONTE TC, WATSON PS. Closure of prosthetic paravalvular mitral regurgitation with the Gianturco-Grifka vascular occlusion device. Catheter Cardiovasc Interv 2001; 54: 234-238.

6. MOORE JD, LASHUS AG, PRIETO LR, DRUMMONDWEBB J, LATSON LA. Transcatheter coil occlusion of perivalvular mitral leaks associated with severe hemolysis. Catheter Cardiovasc Interv 2000;49:64-67.

7. MOSCUCCI M, DEEB GM, BACH D, EAGLE KA, WILLIAMS DM. Coil embolization of a periprosthetic mitral valve leak associated with severe hemolytic anemia. Circulation 2001; 104: E85-E86.

8. BOUDJEMLINE Y, ABDEL-MASSIH T, BONHOEFFER P, PIÉCHAUD JF, AGNOLETTI G, ISERIN F, et al. [Percutaneous closure of a paravalvular mitral regurgitation with Am- 
platzer and coil prostheses]. Arch Mal Coeur Vaiss 2002; 95: 483-486.

9. KORT HW, SHARKEY AM, BALZER DT. Novel use of the Amplatzer duct occluder to close perivalvar leak involving a prosthetic mitral valve. Catheter Cardiovasc Interv 2004; 61: 548-551.

10. YEW G, WILSON NJ. Transcatheter atrial septal defect closure with the Amplatzer septal occluder: Five-year follow-up. Catheter Cardiovasc Interv 2005; 64:193-196.

11. USSIA GP, SCANDURA S, CALAFIORE AM, MANGIAFICO S, MEDURI R, GALASSI AR, et al. Late device dislodgement after percutaneous closure of mitral prosthesis paravalvular leak with Amplatzer muscular ventricular septal defect occlude. Circulation 2007; 115: e208 - 10.

12. ARANZULLA TC, COSGRAVE J, LA CANNA G, MAISANO F, MONTORFANO M, SANGIORGI G, et al. Percutaneous treatment of periprosthetic mitral valve leaks: is it just a futile exercise?. Ann Thorac Surg 2008; 86: 996-8.

13. SHAPIRA Y, HIRSCH R, KORNOWSKI R, HASDAI D, AS SALI A, VATURI M, et al. Percutaneous closure of perivalvular leaks with Amplatzer occluders: feasibility, safety, and short term results. J Heart Valve Dis 2007; 16: 305 - 13.

14. PIECHAUD JF. Percutaneous closure of mitral paravalvular leak. J Interv Cardiol 2003;16: 153-155.

15. HIJAZI ZM. Transcatheter management of paravalvular mitral leaks: far from ideal. Catheter Cardiovasc Interv 2004; 61: 552 - 3 .

16. LATSON LA. Transcatheter closure of paraprosthetic valve leaks after surgical mitral and aortic valve replacements. Expert Rev Cardiovasc Ther 2009; 7: 507 - 14.

17. KURSAKLIOGLU H, BARCIN C, IYISOY A, BAYSAN
O, CELIK T, KOSE S. Percutaneous closure of mitral paravalvular leak via retrograde approach: with use of the Amplatzer duct occlude II and without a wire loop. Tex Heart Inst J 2010; 37: 461 - 4.

18. LIM DS, RAGOSTA M, DENT JM. Percutaneous transthoracic ventricular puncture for diagnostic and interventional catheterization. Catheter Cardiovasc Interv 2008; 71: 915 - 8.

19. LASORDA DM, MOHSIN JC. Percutaneous Closure of Perivalvular Mitral Regurgitation with an Amplatzer Occluder Device in a Patient with Both Prosthetic Mitral and Aortic Valves. J Interven Cardiol 2008; 21: 190-195.

20. SWAANS MJ, POST MC, VAN DER VEN HA, HEIJMEN RH, BUDTS W, TEN BERG JM. Transapical treatment of paravalvular leaks in patients with a logistic euroscore of more than 15\%: Acute and 3-month outcomes of a "proof of concept" study. Catheter Cardiovasc Inter 2011 Jul 29. Doi: 10.1002/ ccd.23264. [Epub ahead of print].

21. YE J, CHEUNG A, LICHTENSTEIN SV, CARERE RG THOMPSON CR, PASUPATI S, et al. Transapical aortic valve implantation in man. J Thorac Cardiovasc Surg 2006; 131: $1194-6$.

22. YE J, CHEUNG A, LICHTENSTEIN SV, PASUPATI S, CARERE RG, THOMPSON CR, et al. Six - month outcome of transapical transcatheter aortic valve implantation in the initial seven patients. European Journal of Cardio - thoracic Surgery 2007; 31: 16-21.

23. CORTÉS M, GARCÍA E, GARCÍA-FERNANDEZ MA, GOMEZ JJ, PEREZ-DAVID E, FERNÁNDEZ-AVILÉS F. Usefulness of Transesophageal Echocardiography in Percutaneous Transcatheter Repairs of Paravalvular Mitral Regurgitation. Am J Cardiol 2008; 101: 382-386. 